\title{
ANALYSIS OF MOVING MESH PARTIAL DIFFERENTIAL EQUATIONS WITH SPATIAL SMOOTHING*
}

\begin{abstract}
WEIZHANG HUANG ${ }^{\dagger}$ AND ROBERT D. RUSSELL ${ }^{\ddagger}$
Abstract. Two moving mesh partial differential equations (MMPDEs) with spatial smoothing are derived based upon the equidistribution principle. This smoothing technique is motivated by the robust moving mesh method of Dorfi and Drury [J. Comput. Phys., 69 (1987), pp. 175-195]. It is shown that under weak conditions the basic property of no node-crossing is preserved by the spatial smoothing, and a local quasi-uniformity property of the coordinate transformations determined by these MMPDEs is proven. It is also shown that, discretizing the MMPDEs using centered finite differences, these basic properties are preserved.
\end{abstract}

Key words. moving mesh PDE, spatial smoothing

AMS subject classifications. 65M50, 65L50, 65N50

PII. S0036142993256441

1. Introduction. For the numerical solution of time-dependent PDEs which involve large solution variations, a variety of moving mesh methods have been shown to gain significant improvements in accuracy and efficiency over conventional fixed mesh methods (e.g., see [GDM81], [AF86], [DD87], [FVZ90], [HGH91], and [HRR94b]). For these methods, mesh equations which involve node speeds are employed to move a mesh (generally having a fixed number of nodes) in such a way that the nodes remain concentrated in regions of rapid variation of the solution. For almost all of them, mesh equations are represented in discrete form.

To facilitate a better understanding of moving mesh equations and to allow for a better investigation of their basic properties, several continuous moving mesh equations (MMPDEs) based upon the equidistribution principle have recently been derived in [HRR94a]. Some basic properties, such as stability and the potential for node-crossing, are also analyzed. While the MMPDEs are derived in such a way that temporal mesh smoothing occurs, a spatial mesh smoothing is also generally necessary, and it is employed when developing practical moving mesh methods based upon these MMPDEs in [HRR94b]. Since the accuracy and error in the solution may depend upon the type of discretization, the quality of the mesh, the treatment of boundary conditions, etc., there is generally no simple relationship between the smoothness of the mesh and the error (cf. [VR92]). Nevertheless, for most problems and most discretization methods, abrupt variations in the mesh will cause a deterioration in the convergence rate and an increase in the error [MTW85]. Moreover, most discrete approximations of spatial differential operators have much larger condition numbers on an abruptly varying mesh than they do on a gradually varying one (e.g., see [SHR96]), and these ill-conditioned approximations may result in stiffness in the time integration for time-dependent problems. It is thus not surprising that mesh

* Received by the editors September 30, 1993; accepted for publication (in revised form) September 12, 1995. This work was supported by the Natural Science and Engineering Research Council of Canada grant OGP-0008781.

http://www.siam.org/journals/sinum/34-3/25644.html

${ }^{\dagger}$ Department of Mathematics and Statistics, Simon Fraser University, Burnaby B.C. V5A 1S6, Canada. Current address: Department of Mathematics, 405 Snow Hall, University of Kansas, Lawrence, KS 66045 (whuang@math.ukans.edu).

${ }^{\ddagger}$ Department of Mathematics and Statistics, Simon Fraser University, Burnaby B.C. V5A 1S6, Canada (rdr@cs.sfu.ca). 
quality has become an increasingly important issue in the field of mesh generation and mesh adaption [MTW85].

The objective of the paper is to introduce and analyze a natural spatial smoothing for the MMPDEs. The spatial smoothing technique is partially motivated by the robust moving mesh method of Dorfi and Drury [DD87] and by the enlightening analysis of it in [VBFZ89]. The spatial smoothing is shown to inherit the no nodecrossing property of the unsmoothed methods and to produce a locally quasi-uniform coordinate transformation (with discrete form corresponding to the well-known local quasi-uniformity property [KN82], which provides a good measure of the smoothness quality of a mesh in one dimension).

An outline of this paper is as follows. In section 2 the MMPDEs with spatial smoothing are derived. The spatial smoothing is incorporated into the mathematical analysis of the MMPDEs and some of their finite difference approximations in sections 3 and 4, respectively. Two numerical examples are provided in section 5 to show the preservation of the local quasi uniformity of the mesh. Section 6 contains conclusions and comments.

2. Formulation of MMPDEs with spatial smoothing. We shall derive in this section our basic MMPDEs which are based upon the equidistribution principle and which also have spatial smoothing.

Let $x$ and $\xi$ denote the physical and computational coordinates, respectively, both of which are without loss of generality assumed to be the unit interval $[0,1]$. A oneto-one coordinate transformation between these domains, which will be determined by an MMPDE, is denoted by

$$
x=x(\xi, t), \quad \xi \in[0,1]
$$

with

$$
x(0, t)=0, \quad x(1, t)=1,
$$

where $t$ denotes time. For a given monitor function $M(x, t)(>0)$ which provides some measure of the computational difficulty in the solution of the underlying physical differential equations, the equidistribution principle can be expressed in differential form (e.g., see [HRR94a]) as

$$
\frac{\partial}{\partial \xi}\left\{M(x(\xi, t), t) \frac{\partial x}{\partial \xi}(\xi, t)\right\}=0 .
$$

Given $M(x, t)$ and an initial transformation $x=x(\xi, 0), x=x(\xi, t)$ can be determined for $t>0$ from (3) and the boundary conditions (2). Since the initial transformation is often chosen as a very smooth function, such as the "uniform transformation" $x(\xi, 0)=$ $\xi$, or as a function determined from $M(x, 0)$, it is realistic to assume that its properties like smoothness are determined by $M(x, t)$. However, for most problems which involve large solution variations, the monitor function is generally fairly nonsmooth in space, and some kind of smoothing of $M(x, t)$ should be employed in (3) in order to make the transformation smooth (see [VBFZ89], [FVZ90], and [HRR94b]). To do this we introduce a PDE in $\xi$ and $t$ which involves an artificial diffusion term for smoothing $M$, viz., we define $\tilde{M}$ to satisfy

$$
\tilde{M}-\lambda^{-2} \Delta \tilde{M}=M
$$


and the boundary conditions

$$
\frac{\partial \tilde{M}}{\partial \xi}(0, t)=\frac{\partial \tilde{M}}{\partial \xi}(1, t)=0 .
$$

Here, $\lambda$ is a positive number and

$$
\Delta=\frac{\partial^{2}}{\partial \xi^{2}}
$$

Denoting by $G$ the restriction of the operator $I-\lambda^{-2} \Delta$ on the space of functions satisfying the boundary conditions (5), the solution of (4) and (5) can be expressed by

$$
\tilde{M}=G^{-1} M .
$$

In the next section we show that $G^{-1}$ exists and analyze the extra smoothness properties of $\tilde{M}$. It is worth noting that the operator $G$ and its inverse $G^{-1}$ commute with the time differentiation. Replacing $M$ in (3) by $\tilde{M}$ gives the "smoother" equidistribution principle

$$
\frac{\partial}{\partial \xi}\left\{\tilde{M}(\xi, t) \frac{\partial x}{\partial \xi}(\xi, t)\right\}=0
$$

or

$$
\frac{\partial}{\partial \xi}\left\{\left(G^{-1} M\right)(\xi, t) \frac{\partial x}{\partial \xi}(\xi, t)\right\}=0 .
$$

As discussed in [HRR94a], a quasi-static form like (9) is an idealized form expressing exact equidistribution, and in practice it can produce nonsmooth trajectories. Mesh equations involving node speeds, or so-called MMPDEs, based upon (9) generally produce much smoother mesh trajectories and are more suited for the numerical solution of time-dependent problems. Following the approach in [HRR94a], we require $(9)$ to be satisfied at the later time $t+\tau(0<\tau \ll 1)$. Expanding the resulting equation in a Taylor series in this relaxation parameter $\tau$ and dropping higher-order terms, we obtain

$$
\frac{\partial}{\partial \xi}\left\{\frac{\partial}{\partial t}\left(\frac{\partial x}{\partial \xi} G^{-1} M\right)\right\}=-\frac{1}{\tau} \frac{\partial}{\partial \xi}\left\{\frac{\partial x}{\partial \xi} G^{-1} M\right\}
$$

or

$$
\frac{\partial}{\partial \xi}\left\{\frac{\partial x}{\partial \xi} \frac{\partial}{\partial t}\left(G^{-1} M\right)+\frac{\partial \dot{x}}{\partial \xi} G^{-1} M\right\}=-\frac{1}{\tau} \frac{\partial}{\partial \xi}\left\{\frac{\partial x}{\partial \xi} G^{-1} M\right\},
$$

where $\dot{x}$ denotes $\left.\frac{\partial x}{\partial t}\right|_{\xi \text { fixed }}$. In actual computation, the term $\frac{\partial}{\partial t}\left(G^{-1} M\right)$ will be complicated and difficult to compute. It may be argued [HRR94a] that it is reasonable to drop this term, giving the simplification

$$
\frac{\partial}{\partial \xi}\left\{\frac{\partial \dot{x}}{\partial \xi} G^{-1} M\right\}=-\frac{1}{\tau} \frac{\partial}{\partial \xi}\left\{\frac{\partial x}{\partial \xi} G^{-1} M\right\} .
$$

This can be regarded as a smooth version of MMPDE 4 in [HRR94a], and similar arguments can be used to show that (10) is asymptotically stable in the sense that its solution satisfies

$$
\frac{\partial}{\partial \xi}\left\{\frac{\partial x}{\partial \xi} G^{-1} M\right\}(\xi, t)=e^{-\frac{t}{\tau}} \frac{\partial}{\partial \xi}\left\{\frac{\partial x}{\partial \xi} G^{-1} M\right\}(\xi, 0) .
$$


Although analogous stability properties have not as yet been proved for (12), numerical experiments indicate that its solution is stable and continues to approximately equidistribute the monitor function for all times if a relatively small value of $\tau$ is used (cf. [HRR94a]).

Thus, one way to obtain a mesh which is smooth in both space and time is to solve

$$
\frac{\partial}{\partial \xi}\left\{\tilde{M} \frac{\partial \dot{x}}{\partial \xi}\right\}=-\frac{1}{\tau} \frac{\partial}{\partial \xi}\left\{\tilde{M} \frac{\partial x}{\partial \xi}\right\}
$$

and (4) together for $x$ and $\tilde{M}$. However, in some respects it does not seem to be a suitable approach since an additional equation (4) is introduced for $\tilde{M}$, and it does not involve time derivatives. Thus, here we shall not consider this method further but introduce an alternative method which is likely to be more efficient and appropriate for time-dependent problems.

In (12), $G^{-1}$ is an integral operator, and discretization produces a dense matrix system. To obtain an alternative form we integrate in $\xi$ to give

$$
\left(G^{-1} M\right)\left(\frac{\partial \dot{x}}{\partial \xi}+\frac{1}{\tau} \frac{\partial x}{\partial \xi}\right)=c(t)
$$

where $c(t)$ is an integral constant, and rewrite this as

$$
M=c(t) G\left(\frac{1}{\frac{\partial \dot{x}}{\partial \xi}+\frac{1}{\tau} \frac{\partial x}{\partial \xi}}\right) .
$$

Now, differentiating with respect to $\xi$, we obtain

$$
\frac{\partial}{\partial \xi}\left\{\frac{G\left(\frac{1}{\frac{\partial \dot{x}}{\partial \xi}+\frac{1}{\tau} \frac{\partial x}{\partial \xi}}\right)}{M}\right\}=0
$$

Since $G$ is defined on the space of functions satisfying $(5)$, we require $x=x(\xi, t)$ to satisfy

$$
\frac{\partial}{\partial \xi}\left(\frac{1}{\frac{\partial \dot{x}}{\partial \xi}+\frac{1}{\tau} \frac{\partial x}{\partial \xi}}\right)=0 \text { at } \xi=0 \text { and } \xi=1
$$

for all positive $\tau$. If $\frac{\partial x}{\partial \xi}>0$ (which will later be shown to be satisfied), then this is equivalent to requiring

$$
\frac{\partial^{2} x}{\partial \xi^{2}}(0, t)=\frac{\partial^{2} x}{\partial \xi^{2}}(1, t)=0 .
$$

Hence, $x(\xi)$ can be determined by solving the smooth MMPDE in the form

$$
\frac{\partial}{\partial \xi}\left\{\frac{\left(I-\lambda^{-2} \Delta\right)\left(\frac{1}{\frac{\partial \dot{x}}{\partial \xi}+\frac{1}{\tau} \frac{\partial x}{\partial \xi}}\right)}{M}\right\}=0
$$

together with the boundary conditions (2) and (18). 
Alternatively, it is instructive to introduce the so-called concentration function

$$
n(\xi, t)=\frac{1}{\frac{\partial x}{\partial \xi}}
$$

and to derive a smooth MMPDE for $x$ written in terms of $n$. If we assume that $\frac{\dot{n}}{n}$ is uniformly bounded for all $\xi$ and $t$, then for small $\tau$

$$
\begin{aligned}
\frac{1}{\frac{\partial \dot{x}}{\partial \xi}+\frac{1}{\tau} \frac{\partial x}{\partial \xi}} & =\frac{\tau n}{1-\frac{\tau \dot{n}}{n}} \\
& =\tau n\left(1+\frac{\tau \dot{n}}{n}+\cdots\right) \\
& =\tau n+\tau^{2} \dot{n}+O\left(\tau^{3}\right) .
\end{aligned}
$$

Substituting (21) into (16) and dropping higher-order terms gives

$$
\frac{\partial}{\partial \xi}\left\{\frac{G\left(\dot{n}+\frac{1}{\tau} n\right)}{M}\right\}=0
$$

where the definition of $G$ requires that $n$ satisfy the boundary conditions

$$
\frac{\partial n}{\partial \xi}(0, t)=\frac{\partial n}{\partial \xi}(1, t)=0
$$

This MMPDE is

$$
\frac{\partial}{\partial \xi}\left\{\frac{\left(I-\lambda^{-2} \Delta\right)\left(\dot{n}+\frac{1}{\tau} n\right)}{M}\right\}=0
$$

or, equivalently,

$$
\frac{\partial}{\partial \xi}\left(\frac{\dot{\hat{n}}}{M}\right)=-\frac{1}{\tau} \frac{\partial}{\partial \xi}\left(\frac{\hat{n}}{M}\right)
$$

where

$$
\hat{n}=G n .
$$

Note that both (19) and (25) are fourth-order (in space) differential equations for $x$ requiring four boundary conditions. As will be seen, a centered finite difference discretization of (25) gives exactly the moving finite difference method developed by Dorfi and Drury in [DD87] (see section 4). Indeed, their scheme provides the original motivation for our introducing smoothing with the diffusion term in (4). However, (25) (or (19)) is more general and useful due to its differential form, and it can be solved by any of the standard numerical methods, such as a finite element, collocation, or spectral method. Furthermore, the role of $\tau$ is more transparent using this continuous MMPDE approach.

Notice that the MMPDE (25) is only slightly more complicated than the (nonsmoothed) MMPDEs in [HRR94a]. In the same way that MMPDE6 is obtained from MMPDE4 in [HRR94a], a simple alternative MMPDE can be obtained from (25) by taking $M=1$ on the left-hand side, giving

$$
\frac{\partial}{\partial \xi}(\dot{\hat{n}})=-\frac{1}{\tau} \frac{\partial}{\partial \xi}\left(\frac{\hat{n}}{M}\right) .
$$


The "smoothed" equidistribution principle (8) can be rewritten as

$$
\frac{\partial}{\partial \xi}\left(\frac{\hat{n}}{M}\right)=0
$$

Therefore, the right-hand side term

$$
-\frac{1}{\tau} \frac{\partial}{\partial \xi}\left(\frac{\hat{n}}{M}\right)
$$

in $(25)$ and (27) can be interpreted as a source for the mesh movement, as a stabilizing term, or as a mechanism to pull the mesh back toward equidistribution, in the analogous manner to the nonsmooth MMPDE context in [HRR94a].

In actual computation, the MMPDE is normally discretized at $N$ equally spaced points

$$
\xi_{i}=i h, \quad i=0,1, \ldots, N,
$$

where $h=\frac{1}{N}$. Then, a mesh $x_{i}=x\left(\xi_{i}, t\right), i=0,1, \ldots, N$, is obtained by solving the resulting discrete mesh equations.

The spatial smoothing technique in [HRR94b] which uses a certain average of monitor function values at $2 p+1$ adjacent mesh points can be interpreted with our approach here. It is not difficult to see that if the value of $\lambda$ is properly chosen, this average is closely related to replacing $G^{-1}$ by the approximation

$$
I+\lambda^{-2} \Delta+\left(\lambda^{-2} \Delta\right)^{2}+\cdots+\left(\lambda^{-2} \Delta\right)^{p}
$$

and using centered differences to approximate $\Delta$ (e.g., $\Delta M_{i} \approx \frac{M_{i+1}-2 M_{i}+M_{i-1}}{h^{2}}$ ).

It is generally necessary that the mesh has no node-crossings and varies gradually in space. The first condition is guaranteed if

$$
x_{i+1}(t)-x_{i}(t)>0, i=0,1, \ldots, N-1,
$$

and to satisfy the second condition the natural requirement is that the mesh be locally quasi uniform, that is,

$$
\frac{1}{K} \leq \frac{x_{i+1}-x_{i}}{x_{i}-x_{i-1}} \leq K, i=1,2, \ldots, N-1,
$$

for some fixed constant $K>1$. Continuous analogues of these are the so-called no node-crossing condition (or mesh consistency condition)

$$
\frac{\partial x}{\partial \xi}>0
$$

and the local quasi-uniformity condition

$$
\left|\frac{\frac{\partial^{2} x}{\partial \xi^{2}}}{\frac{\partial x}{\partial \xi}}\right| \leq \lambda
$$

for the mesh transformation.

The discrete equivalent of (20) is

$$
n_{i+\frac{1}{2}}(t)=\frac{h}{x_{i+1}(t)-x_{i}(t)} .
$$


This is the familiar mesh concentration function used in [DD87] and frequently appearing in the literature on moving mesh methods. In terms of the concentration function $n(\xi, t)$, no node-crossing and local quasi uniformity can be expressed by

$$
n(\xi, t)>0
$$

and

$$
\left|\frac{\frac{\partial n}{\partial \xi}}{n}\right| \leq \lambda
$$

In the next section, we shall show that the solutions of MMPDEs (19) and (25) have properties (34) and (35) or (37) and (38), respectively. After that, the finite difference discretization of these MMPDEs will be shown to inherit these properties in the forms (32) and (33).

3. Analysis of MMPDEs. The spatial smoothing properties of the operator $G$ are described in the following simple lemma, whose proof is given for completeness.

LEMMA 3.1. Suppose that $f(\xi)$ is a function defined on $[0,1]$ satisfying

$$
0<\alpha \leq f(\xi) \leq \beta<\infty \quad \text { for all } \xi \in[0,1] .
$$

Then, the solution of

$$
\left\{\begin{array}{l}
G v \equiv\left(I-\lambda^{-2} \Delta\right) v=f, \quad 0<\xi<1 \\
\frac{d v}{d \xi}(0)=\frac{d v}{d \xi}(1)=0
\end{array}\right.
$$

exists for all $\lambda>0$ (therefore, $G$ has an inverse), and $v(\xi)=G^{-1} f$ has the following properties:

(i) $v$ also satisfies

$$
0<\alpha \leq v(\xi) \leq \beta<\infty \quad \text { for all } \xi \in[0,1] .
$$

(ii) $v$ satisfies the smoothness condition

$$
\left|\frac{d v}{v}\right| \leq \lambda
$$

Proof. Letting $w:=\frac{d v}{d \xi},(40)$ can be rewritten as

$$
\left\{\begin{array}{l}
\frac{d v}{d \xi}=w \\
\frac{d w}{d \xi}=\lambda^{2} v-\lambda^{2} f
\end{array}\right.
$$

with $w(0)=w(1)=0$. Writing the solution of (42) in the form

$$
\left\{\begin{array}{l}
v=c_{1}(\xi) e^{\lambda \xi}+c_{2}(\xi) e^{-\lambda \xi} \\
w=\lambda c_{1}(\xi) e^{\lambda \xi}-\lambda c_{2}(\xi) e^{-\lambda \xi}
\end{array}\right.
$$

leads to

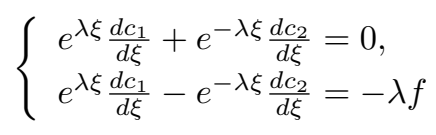


which gives

$$
\left\{\begin{array}{l}
c_{1}(\xi)=-\frac{\lambda}{2} \int_{0}^{\xi} e^{-\lambda \xi} f(\xi) d \xi+c_{3} \\
c_{2}(\xi)=+\frac{\lambda}{2} \int_{0}^{\xi} e^{\lambda \xi} f(\xi) d \xi+c_{4}
\end{array}\right.
$$

where $c_{3}$ and $c_{4}$ are integral constants. Substituting (44) into (43), we obtain the solution

$$
\left\{\begin{array}{l}
v=e^{\lambda \xi}\left(c_{3}-\frac{\lambda}{2} \int_{0}^{\xi} e^{-\lambda \xi} f(\xi) d \xi\right)+e^{-\lambda \xi}\left(c_{4}+\frac{\lambda}{2} \int_{0}^{\xi} e^{\lambda \xi} f(\xi) d \xi\right), \\
w=\lambda e^{\lambda \xi}\left(c_{3}-\frac{\lambda}{2} \int_{0}^{\xi} e^{-\lambda \xi} f(\xi) d \xi\right)-\lambda e^{-\lambda \xi}\left(c_{4}+\frac{\lambda}{2} \int_{0}^{\xi} e^{\lambda \xi} f(\xi) d \xi\right) .
\end{array}\right.
$$

From the boundary conditions,

$$
\begin{aligned}
& c_{3}=c_{4}, \\
& c_{4}=\frac{\lambda}{2\left(e^{\lambda}-e^{-\lambda}\right)}\left(e^{\lambda} \int_{0}^{1} e^{-\lambda \xi} f(\xi) d \xi+e^{-\lambda} \int_{0}^{1} e^{\lambda \xi} f(\xi) d \xi\right) .
\end{aligned}
$$

Defining

$$
g=\frac{1}{e^{\lambda}-e^{-\lambda}}\left(e^{\lambda} \int_{0}^{1} e^{-\lambda \xi} f(\xi) d \xi+e^{-\lambda} \int_{0}^{1} e^{\lambda \xi} f(\xi) d \xi\right)
$$

we obtain

$$
\left\{\begin{array}{l}
v(\xi)=\frac{\lambda e^{\lambda \xi}}{2}\left(g-\int_{0}^{\xi} e^{-\lambda \xi} f(\xi) d \xi\right)+\frac{\lambda e^{-\lambda \xi}}{2}\left(g+\int_{0}^{\xi} e^{\lambda \xi} f(\xi) d \xi\right), \\
\frac{d v}{d \xi}=\frac{\lambda^{2} e^{\lambda \xi}}{2}\left(g-\int_{0}^{\xi} e^{-\lambda \xi} f(\xi) d \xi\right)-\frac{\lambda^{2} e^{-\lambda \xi}}{2}\left(g+\int_{0}^{\xi} e^{\lambda \xi} f(\xi) d \xi\right) .
\end{array}\right.
$$

Since $g>0$ and

$$
\begin{aligned}
g-\int_{0}^{\xi} & e^{-\lambda \xi} f(\xi) d \xi \\
& =\frac{1}{e^{\lambda}-e^{-\lambda}}\left(e^{\lambda} \int_{\xi}^{1} e^{-\lambda \xi} f(\xi) d \xi+e^{-\lambda} \int_{0}^{1} e^{\lambda \xi} f(\xi) d \xi+e^{-\lambda} \int_{0}^{\xi} e^{\lambda \xi} f(\xi) d \xi\right) \\
& >0
\end{aligned}
$$

(i) follows. Conclusion (ii) follows easily from

$$
\begin{aligned}
\left|\frac{d v}{d \xi}\right| & \leq \frac{\lambda^{2} e^{\lambda \xi}}{2}\left(g-\int_{0}^{\xi} e^{-\lambda \xi} f(\xi) d \xi\right)+\frac{\lambda^{2} e^{-\lambda \xi}}{2}\left(g+\int_{0}^{\xi} e^{\lambda \xi} f(\xi) d \xi\right) \\
& =\lambda v .
\end{aligned}
$$

To obtain the main results about the solutions of MMPDEs (19) and (25), we shall need two mild assumptions.

Assumption 3.1. The monitor function $M(x, t)$ satisfies

$$
0<\alpha_{0} \leq M(x, t) \leq \alpha_{1}<\infty
$$

for all $x \in[0,1]$ and $0 \leq t \leq T$.

Assumption 3.2. The initial coordinate transformation satisfies

$$
0<\beta_{0} \leq \frac{\partial x}{\partial \xi}(\xi, 0) \leq \beta_{1}<\infty
$$

for all $\xi \in[0,1]$. 
Note that using the arclength monitor function, for example, (48) basically requires that the first derivative of the solution to the physical PDE remain bounded for all time.

THEOREM 3.1. If Assumptions 3.1 and 3.2 hold, then the solution $n(\xi, t)=\frac{1}{\frac{\partial x}{\partial \xi}}$ of (25) for which (2) and (23) are satisfied has the following properties:

(i) For all $\xi \in[0,1]$ and $0 \leq t \leq T$,

$$
e^{-\frac{t}{\tau}} n(\xi, 0) \leq n(\xi, t) \leq e^{-\frac{t}{\tau}} n(\xi, 0)+\frac{\alpha_{1}}{\alpha_{0}},
$$

so this solution of MMPDE (25) satisfies the no node-crossing condition.

(ii) If the initial transformation satisfies

$$
\left|\frac{\frac{\partial n}{\partial \xi}(\xi, 0)}{n(\xi, 0)}\right| \leq \lambda \text { for all } \xi \in[0,1],
$$

then for all $\xi \in[0,1]$ and $0 \leq t \leq T$,

$$
\left|\frac{\frac{\partial n}{\partial \xi}(\xi, t)}{n(\xi, t)}\right| \leq \lambda ;
$$

i.e., an initial local quasi-uniformity condition (defined implicitly through $n(\xi, 0)$ ) gives local quasi uniformity for all times. isfy

$$
\frac{\left(1-e^{-\lambda}\right) e^{-\lambda \xi}}{\lambda} \leq n(\xi, t) \leq \frac{\left(e^{\lambda}-1\right) e^{\lambda \xi}}{\lambda} .
$$

Proof. Define $t^{*}$ by

$$
t^{*}:=\sup \left\{\tilde{t} \mid \frac{\partial x}{\partial \xi}(\xi, t)>0 \text { for all } 0 \leq t \leq \tilde{t} \text { and } \xi \in[0,1]\right\} ;
$$

so from Assumption 3.2, $t^{*}>0$. For $0 \leq t<t^{*}$, from (25)

$$
\frac{\partial}{\partial \xi}\left(\frac{\dot{\hat{n}}+\frac{1}{\tau} \hat{n}}{M}\right)=0 .
$$

Integrating in $\xi$, we have

$$
\dot{\hat{n}}+\frac{1}{\tau} \hat{n}=c(t) M,
$$

where $\tilde{M}=G^{-1} M$ and $c(t)$ is the integration constant. Thus,

$$
\dot{n}+\frac{1}{\tau} n=c(t) \tilde{M},
$$

so

$$
\frac{\partial \dot{x}}{\partial \xi}-\frac{1}{\tau} \frac{\partial x}{\partial \xi}=-c(t) \tilde{M}\left(\frac{\partial x}{\partial \xi}\right)^{2}
$$


From (2)

$$
c(t)=\left\{\tau \int_{0}^{1} \tilde{M}\left(\frac{\partial x}{\partial \xi}\right)^{2} d \xi\right\}^{-1} .
$$

Since $\frac{\partial x}{\partial \xi}>0$ for $0 \leq t<t^{*}$ and since $\tilde{M}$ is a positive function satisfying (41) from Lemma 3.1 and Assumption 3.1, we have

$$
c(t)>0 \text { for } 0 \leq t<t^{*} .
$$

Integrating (55) in time gives

$$
n(\xi, t)=e^{-\frac{t}{\tau}}\left(n(\xi, 0)+\int_{0}^{t} c(\tilde{t}) e^{\frac{\tilde{t}}{\tau}} \tilde{M}(\xi, \tilde{t}) d \tilde{t}\right)
$$

so that

$$
n(\xi, t) \geq e^{-\frac{t}{\tau}} n(\xi, 0) \text { for } 0 \leq t<t^{*} .
$$

The condition $x(1, t)=1$ implies that

$$
\int_{0}^{1} \frac{1}{n(\xi, t)} d \xi=1
$$

hence, from (59)

$$
\int_{0}^{1} \frac{d \xi}{n(\xi, 0)+\int_{0}^{t} c(\tilde{t}) e^{\frac{\tilde{t}}{\tau}} \tilde{M}(\xi, \tilde{t}) d \tilde{t}}=e^{-\frac{t}{\tau}}
$$

From Assumptions 3.1 and 3.2 we have

$$
e^{-\frac{t}{\tau}} \leq \frac{1}{\frac{1}{\beta_{1}}+\alpha_{0} \int_{0}^{t} c(\tilde{t}) e^{\frac{\tilde{t}}{\tau}} d \tilde{t}}
$$

or

$$
\frac{e^{\frac{t}{\tau}}}{\alpha_{0}} \geq \int_{0}^{t} c(\tilde{t}) e^{\frac{\tilde{t}}{\tau}} d \tilde{t}
$$

Now, (59) and (62) imply

$$
\begin{aligned}
n(\xi, t) & \leq e^{-\frac{t}{\tau}}\left(n(\xi, 0)+\alpha_{1} \int_{0}^{t} c(\tilde{t}) e^{\frac{\tilde{t}}{\tau}} d \tilde{t}\right) \\
& \leq e^{-\frac{t}{\tau}}\left(n(\xi, 0)+\frac{\alpha_{1} e^{\frac{t}{\tau}}}{\alpha_{0}}\right) \\
& =e^{-\frac{t}{\tau}} n(\xi, 0)+\frac{\alpha_{1}}{\alpha_{0}} .
\end{aligned}
$$

Thus, we have obtained (50) for $0 \leq t<t^{*}$. From the continuity of $n(\xi, t)$, (50) is also true for $t=t^{*}$. If $t^{*}<T$, then there exists at least one value of $\xi$, say $\xi^{*}$, such that $\frac{\partial x}{\partial \xi}\left(\xi^{*}, t^{*}\right)=0$. Since from (50) this is impossible, conclusion (i) holds. 
From Assumption 3.1 and Lemma 3.1, $\tilde{M}$ is positive and satisfies (41). Differentiating (59) with respect to $\xi$ and using (58), we have

$$
\begin{aligned}
\left|\frac{\partial n}{\partial \xi}(\xi, t)-e^{-\frac{t}{\tau}} \frac{\partial n}{\partial \xi}(\xi, 0)\right| & \leq e^{-\frac{t}{\tau}} \int_{0}^{t} c(\tilde{t}) e^{\frac{\tilde{t}}{\tau}}\left|\frac{\partial \tilde{\mu}}{\partial \xi}\right| \tilde{M} d \tilde{t} \\
& \leq \lambda e^{-\frac{t}{\tau}} \int_{0}^{t} c(\tilde{t}) e^{\frac{\tilde{t}}{\tau}} \tilde{M} d \tilde{t} \\
& =\lambda\left(n(\xi, t)-e^{-\frac{t}{\tau}} n(\xi, 0)\right) .
\end{aligned}
$$

Inequality (63), together with (51), gives conclusion (ii).

Conclusion (iii) can be shown to follow from conclusion (ii) using the boundary conditions (2).

Similarly, we have the following theorem for MMPDE (19).

THEOREM 3.2. If Assumptions 3.1 and 3.2 hold, then the solution of (19) with boundary conditions (2) and (18) has the following properties:

(i) For all $\xi \in[0,1]$ and $0 \leq t \leq T$,

$$
e^{-\frac{t}{\tau}} \frac{\partial x}{\partial \xi}(\xi, 0) \leq \frac{\partial x}{\partial \xi}(\xi, t) \leq e^{-\frac{t}{\tau}} \frac{\partial x}{\partial \xi}(\xi, 0)+\frac{\alpha_{1}}{\alpha_{0}} ;
$$

i.e., the no node-crossing condition holds.

(ii) If the initial transformation satisfies

$$
\left|\frac{\frac{\partial^{2} x}{\partial \xi^{2}}(\xi, 0)}{\frac{\partial x}{\partial \xi}(\xi, 0)}\right| \leq \lambda \text { for all } \xi \in[0,1],
$$

then this local quasi-uniformity condition

$$
\left|\frac{\frac{\partial^{2} x}{\partial \xi^{2}}(\xi, t)}{\frac{\partial x}{\partial \xi}(\xi, t)}\right| \leq \lambda
$$

holds for all $\xi \in[0,1]$ and $0 \leq t \leq T$.

(iii) Furthermore, if (65) holds, then

$$
\frac{\lambda e^{-\lambda \xi}}{1-e^{-\lambda}} \leq \frac{\partial x}{\partial \xi}(\xi, t) \leq \frac{\lambda e^{\lambda \xi}}{e^{\lambda}-1} .
$$

4. Finite difference discretization. In the previous section, we have proved that under mild assumptions the solutions of continuous MMPDEs (19) and (25) have the important properties of no node-crossing and local quasi uniformity expressed by (34) and (35) or (37) and (38), respectively. Here, we shall show that discretization methods satisfy discrete equivalents of these properties. For simplicity, we only consider a three-point centered difference method for the discretization.

We introduce a mesh variable $y$ defined by

$$
y_{i+\frac{1}{2}}:=\frac{h}{\dot{x}_{i+1}-\dot{x}_{i}+\frac{1}{\tau}\left(x_{i+1}-x_{i}\right)}, \quad i=0,1, \ldots, N-1,
$$

for MMPDE (19) and by

$$
y_{i+\frac{1}{2}}:=-\frac{h\left(\dot{x}_{i+1}-\dot{x}_{i}\right)}{\left(x_{i+1}-x_{i}\right)^{2}}+\frac{1}{\tau} \frac{h}{x_{i+1}-x_{i}}, \quad i=0,1, \ldots, N-1,
$$


for MMPDE (24) (or (25)). For the uniform computational mesh (30) and $x_{i}:=$ $x\left(\xi_{i}, t\right)$, let

$$
z_{i+\frac{1}{2}}:=y_{i+\frac{1}{2}}-\frac{1}{\lambda^{2} h^{2}}\left(y_{i+\frac{3}{2}}-2 y_{i+\frac{1}{2}}+y_{i-\frac{1}{2}}\right), \quad i=0,1, \ldots, N-1 .
$$

The centered finite difference equations for both MMPDEs (19) and (25) can be expressed by

$$
\frac{z_{i+\frac{1}{2}}}{M_{i+\frac{1}{2}}}=\frac{z_{i-\frac{1}{2}}}{M_{i-\frac{1}{2}}}, \quad i=1,2, \ldots, N-1,
$$

where $M_{i+\frac{1}{2}}=M\left(x_{i+\frac{1}{2}}, t\right)$. Furthermore, we use

$$
y_{-\frac{1}{2}}=y_{\frac{1}{2}}, \quad y_{N+\frac{1}{2}}=y_{N-\frac{1}{2}}
$$

as approximations to the boundary conditions (17) or (23) and

$$
x_{0}=0 \quad \text { and } \quad x_{N}=1
$$

for (2).

We have the following discrete analogue to Lemma 3.1.

LEMMA 4.1. Suppose that $f(\xi)$ is a function defined on $[0,1]$ satisfying (39). Then, the solution of the difference equations

$$
\left\{\begin{array}{l}
v_{i+\frac{1}{2}}-\frac{1}{\lambda^{2} h^{2}}\left(v_{i+\frac{3}{2}}-2 v_{i+\frac{1}{2}}+v_{i-\frac{1}{2}}\right)=f_{i+\frac{1}{2}}, \quad i=0,1, \ldots, N-1, \\
v_{-\frac{1}{2}}=v_{\frac{1}{2}}, \quad v_{N+\frac{1}{2}}=v_{N-\frac{1}{2}}
\end{array}\right.
$$

has the following properties:

(i) For $i=0,1, \ldots, N-1$,

$$
\alpha \leq \min _{j=0, \ldots, N-1} f_{j+\frac{1}{2}} \leq v_{i+\frac{1}{2}} \leq \max _{j=0, \ldots, N-1} f_{j+\frac{1}{2}} \leq \beta .
$$

(ii) For $i=1,2, \ldots, N-1$,

$$
\nu \leq \frac{v_{i+\frac{1}{2}}}{v_{i-\frac{1}{2}}} \leq \nu^{-1}
$$

where

$$
\nu=\frac{\sqrt{1+4 \gamma}-1}{\sqrt{1+4 \gamma}+1}
$$

is one of the roots of the equation $-\gamma \nu^{2}+(1+2 \gamma) \nu-\gamma=0$, where

$$
\gamma=\frac{1}{\lambda^{2} h^{2}} .
$$

Proof. It is not difficult to verify that the solution of (74) is given by

$$
\begin{aligned}
v_{i+\frac{1}{2}}= & \frac{1}{\left(1-\nu^{2 N}\right) \sqrt{1+4 \gamma}} \sum_{j=0}^{N-1} f_{j+\frac{1}{2}} \\
& \times\left[\left(\nu^{2 N+j+\frac{1}{2}}+\nu^{2 N-j-\frac{1}{2}}\right) \nu^{-i-\frac{1}{2}}+\left(\nu^{j+\frac{1}{2}}+\nu^{-j-\frac{1}{2}}\right) \nu^{i+\frac{1}{2}}+\left(1-\nu^{2 N}\right) \nu^{|i-j|}\right] .
\end{aligned}
$$


The conclusions can now be easily obtained using (79) by noticing that (80)

$$
\begin{aligned}
& \frac{1}{\left(1-\nu^{2 N}\right) \sqrt{1+4 \gamma}} \\
& \times \sum_{j=0}^{N-1}\left[\left(\nu^{2 N+j+\frac{1}{2}}+\nu^{2 N-j-\frac{1}{2}}\right) \nu^{-i-\frac{1}{2}}+\left(\nu^{j+\frac{1}{2}}+\nu^{-j-\frac{1}{2}}\right) \nu^{i+\frac{1}{2}}+\left(1-\nu^{2 N}\right) \nu^{|i-j|}\right] \\
& =1
\end{aligned}
$$

for $i=0,1, \ldots, N-1$.

The discrete analogue to Assumption 3.2 is as follows.

Assumption $3.2^{\prime}$. The initial mesh satisfies

$$
0<\beta_{0} \leq \frac{x_{i+1}(0)-x_{i}(0)}{h} \leq \beta_{1}<\infty, \quad i=0,1, \ldots, N-1 .
$$

Using Lemma 4.1 and following the approach in the proof of Theorem 3.1, the next two theorems can be proven.

THEOREM 4.1. If Assumptions 3.1 and $3.2^{\prime}$ hold, then the solution of (71), (72), and (73) using (69) and (70) has the following properties:

(i) For $i=0,1, \ldots, N-1$ and $0 \leq t \leq T$,

$$
e^{-\frac{t}{\tau}} n_{i+\frac{1}{2}}(0) \leq n_{i+\frac{1}{2}}(t) \leq e^{-\frac{t}{\tau}} n_{i+\frac{1}{2}}(0)+\frac{\alpha_{1}}{\alpha_{0}},
$$

where

$$
n_{i+\frac{1}{2}}(t)=\frac{h}{x_{i+1}-x_{i}} .
$$

(ii) If the initial mesh is locally quasi uniform

$$
\nu \leq \frac{x_{i+1}(0)-x_{i}(0)}{x_{i}(0)-x_{i-1}(0)} \leq \nu^{-1}, \quad i=1,2, \ldots, N-1,
$$

then the meshes are locally quasi uniform for all $0 \leq t \leq T$, viz.,

$$
\nu \leq \frac{x_{i+1}(t)-x_{i}(t)}{x_{i}(t)-x_{i-1}(t)} \leq \nu^{-1}, \quad i=1,2, \ldots, N-1 .
$$

(iii) If the initial mesh is locally quasi uniform, then

$$
\frac{1-\nu}{1-\nu^{N}} \nu^{i} \leq x_{i+1}(t)-x_{i}(t) \leq \frac{1-\nu^{-1}}{1-\nu^{-N}} \nu^{-i}, \quad i=0,1, \ldots, N-1 .
$$

THEOREM 4.2. If Assumptions 3.1 and $3.2^{\prime}$ hold, then the solution of (71), (72), and (73) using (68) and (70) has the following properties:

(i) For $i=0,1, \ldots, N-1$ and $0 \leq t \leq T$,

$$
e^{-\frac{t}{\tau}} \frac{x_{i+1}(0)-x_{i}(0)}{h} \leq \frac{x_{i+1}(t)-x_{i}(t)}{h} \leq e^{-\frac{t}{\tau}} \frac{x_{i+1}(0)-x_{i}(0)}{h}+\frac{\alpha_{1}}{\alpha_{0}} .
$$

(ii) If the initial mesh is locally quasi uniform

$$
\nu \leq \frac{x_{i+1}(0)-x_{i}(0)}{x_{i}(0)-x_{i-1}(0)} \leq \nu^{-1}, \quad i=1,2, \ldots, N-1,
$$


then the meshes are locally quasi uniform for all $0 \leq t \leq T$, viz.,

$$
\nu \leq \frac{x_{i+1}(t)-x_{i}(t)}{x_{i}(t)-x_{i-1}(t)} \leq \nu^{-1}, \quad i=1,2, \ldots, N-1 .
$$

(iii) If the initial mesh is locally quasi uniform, then

$$
\frac{1-\nu}{1-\nu^{N}} \nu^{i} \leq x_{i+1}(t)-x_{i}(t) \leq \frac{1-\nu^{-1}}{1-\nu^{-N}} \nu^{-i}, \quad i=0,1, \ldots, N-1 .
$$

We conclude this section with two observations about the discrete equations (71), (72), and (73) using (69) and (70). First, it is easy to verify that the mesh equation is exactly the one used in the Dorfi and Drury method if the parameter $\lambda$ is chosen such that $\gamma=\tilde{\gamma}(1+\tilde{\gamma})$. Second, similar results to those in Theorem 4.1 have been obtained in [VBFZ89]. The results in Theorem 4.1 are somewhat stronger than those in [VBFZ89] since we obtain conclusions (ii) and (iii) without requiring that (71) be satisfied initially. More importantly, we interpret the method as a discretization which inherits certain fundamental properties of a continuous transformation defined through the MMPDE (25).

5. Numerical examples. The capabilities of moving mesh methods have been amply demonstrated elsewhere, so the MMPDEs are applied here to two simple problems: a problem with solutions having derivative singularities and the well-known Burgers equation with a smooth initial solution. The physical equations are first transformed into the computational coordinate and then discretized on the uniform mesh (30) by centered finite differences. The resulting finite difference equations, together with the difference equations for MMPDE (19) or (25) (see (79)-(83)) and the corresponding boundary conditions, constitute an ODE system which determines the physical solution $\left\{u_{i}(t)\right\}_{i=0}^{N}$ and the mesh $\left\{x_{i}(t)\right\}_{i=0}^{N}$. The parameter $\lambda$ in the spatial smoothing process is determined by choosing $\gamma$ in (78) as

$$
\gamma=\tilde{\gamma}(\tilde{\gamma}+1)
$$

where the values of $\tilde{\gamma}$ will be given. Notice that $\tilde{\gamma}=0$ corresponds to the case of no spatial smoothing.

This semidiscrete system is integrated in time by using the BDF code DASSL [Pet82], with approximate Jacobians being computed by the code internally via finite differences. For all computations the absolute and relative local time stepping error tolerances (in a root-mean-square norm) are taken as

$$
\text { atol }=r t o l=10^{-7} \text {. }
$$

The initial mesh is chosen as a uniform mesh and the value of the temporal smoothing parameter is taken as $\tau=10^{-3}$. Note that DASSL normally requires $\left\{x_{i}(0), u_{i}(0)\right\}$ and $\left\{\dot{x}_{i}(0), \dot{u}_{i}(0)\right\}$ to be consistent. Since $\left\{\dot{x}_{i}(0), \dot{u}_{i}(0)\right\}$ are unknown DASSL computes them internally. This often requires very small time stepsizes at the beginning of the time integration, and DASSL can sometimes fail altogether to find them. In the results presented, we measure the level of local quasi uniformity

$$
L(t)=\max \left\{\frac{x_{i+1}(t)-x_{i}(t)}{x_{i}(t)-x_{i-1}(t)}, \frac{x_{i}(t)-x_{i-1}(t)}{x_{i+1}(t)-x_{i}(t)}\right\}
$$

and the level of global quasi uniformity

$$
G(t)=\frac{\max \left\{x_{i+1}(t)-x_{i}(t)\right\}}{\min \left\{x_{i+1}(t)-x_{i}(t)\right\}} .
$$


All computations are performed on a Silicon Graphics Indy workstation with a double precision algorithm.

Example 5.1. A problem with solutions having derivative singularities. Our first example is

$$
\begin{aligned}
u_{t}=u_{x x}+f(x, t), & x \in(0,1), \quad 0<t \leq 1, \\
u(0, t)=u(1, t)=0, & 0<t \leq 1, \\
u(x, 0)=0, & x \in[0,1]
\end{aligned}
$$

where $f(x, t)$ is chosen such that the exact solution is

$$
u(x, t)=t\left(x^{\alpha}-x\right), \quad \alpha>\frac{1}{2} .
$$

The parameter $\alpha$ is assumed to be a noninteger number, so that the solution (96) has a derivative singularity at $x=0$, which models the singularity caused by corners of the domain in two-dimensional problems. This type of singularity is thoroughly studied in [GB86] for $h, p$, and $h-p$ versions of the finite element method in one dimension, and a class of optimal meshes with respect to solution error in several norms is presented.

Interestingly, such optimal meshes can also be obtained using the equidistribution principle (3). Specifically, for the time-independent problem Carey and Dinh [CD85] show that if the monitor function is chosen as

$$
M=\left[u^{(p+1)}\right]^{2 /[2(p+1-m)+1]},
$$

where the approximate solution $u$ is a polynomial of degree $p$ in each subinterval, then the solution error $e$ is minimized in the $H^{m}$ seminorm $|\bullet|_{m}\left(\right.$ where $\left.|e|_{m}^{2}:=\int_{0}^{1}\left[e^{(m)}\right]^{2} d x\right)$ and can be bounded by

$$
|e|_{m}^{2} \leq \frac{1}{(\pi N)^{2(p+1-m)}} \int_{0}^{1} \frac{\left[u^{(p+1)}\right]^{2}}{\left[\xi^{\prime}\right]^{2(p+1-m)}} d x\left(1+O\left(\Delta x_{\max }\right)\right),
$$

where $\xi^{\prime}$ denotes $\frac{\partial \xi}{\partial x}$. It is straightforward to show from (3) that the optimal coordinate transformation (mesh) for the solution (96) is

$$
x=\xi^{[2(p+1-m)+1] /[2 \alpha-2 m+1]},
$$

and for $m=1$, (99) is precisely the so-called optimal radical mesh of Gui and Babuška [GB86].

The three-point centered finite difference approximation can be regarded as a polynomial approximation of degree $p=1$ in $x$ (cf. [CD85]). If the energy norm

$$
|e|_{E}(t):=\left\{\int_{0}^{1}\left|\frac{\partial e}{\partial x}\right|^{2} d x\right\}^{1 / 2}
$$

is used (i.e., $m=1$ ), then the optimal monitor function is

$$
M=\left[u_{x x}\right]^{2 / 3},
$$


TABLE 1

Numerical results for Example 5.1 with $\alpha=0.7 . L, G$, and $|e|_{E}$ are evaluated at $t=1$.

\begin{tabular}{ccccccc}
\hline \hline & $N$ & $L$ & $G$ & $|e|_{E}$ & Order & CPU (second) \\
\hline \hline No smoothing & 10 & 2.06 & $9.29 \mathrm{E} 1$ & 0.168 & & 1.1 \\
$\tilde{\gamma}=0$ & 20 & 2.06 & $2.42 \mathrm{E} 3$ & $8.71 \mathrm{E}-2$ & 0.95 & 3.0 \\
& 40 & 2.06 & $1.10 \mathrm{E} 5$ & $4.12 \mathrm{E}-2$ & 1.08 & 8.1 \\
& 80 & 2.06 & $6.94 \mathrm{E} 6$ & $1.89 \mathrm{E}-2$ & 1.12 & 37.4 \\
\hline \hline With smoothing & 10 & 1.42 & $1.28 \mathrm{E} 1$ & 0.229 & & 1.1 \\
$\tilde{\gamma}=1$ & 20 & 1.42 & $2.12 \mathrm{E} 2$ & 0.127 & 0.85 & 2.1 \\
& 40 & 1.42 & $9.23 \mathrm{E} 3$ & $5.81 \mathrm{E}-2$ & 1.13 & 6.6 \\
& 80 & 1.42 & $6.74 \mathrm{E} 5$ & $2.44 \mathrm{E}-2$ & 1.25 & 17.4 \\
\hline \hline
\end{tabular}
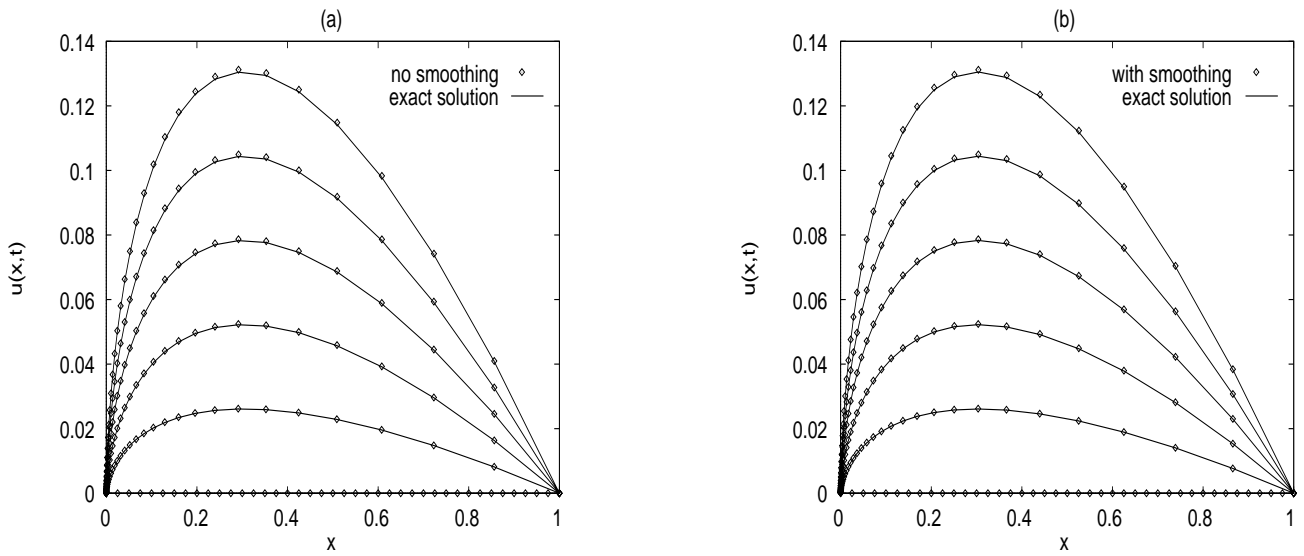

FiG. 1. Example 5.1. Solutions at different times $t=0.0,0.2,0.4,0.6,0.8$, and 1.0. $N=40$ is used.

the corresponding optimal mesh transformation is

$$
x=\xi^{3 /(2 \alpha-1)},
$$

and the error is bounded by

$$
|e|_{E} \leq O\left(\frac{1}{N}\right) .
$$

Numerical results obtained with MMPDE (25) and the monitor function (101) are presented for $\alpha=0.7$ in Table 1 and Figures 1-3. Table 1 shows the error, $L$, $G$, and CPU time for the moving mesh method with and without smoothing. We can easily see from this table the first-order convergence for the energy norm error in both cases, which is consistent with (103). Figure 2 shows the rapid convergence of the computed mesh to the continuous, optimal mesh (102).

For this problem, the monitor function (101) changes gradually. As a consequence, the MMPDE produces a fairly smooth mesh even without spatial smoothing. This can be seen clearly in Table 1 . In fact, the function $L(t)$, the measurement of local quasi uniformity of the mesh, has a value of about two even without smoothing. Therefore, it can be expected that the spatial smoothing will not play a significant role in improving accuracy, and it even reduces the accuracy slightly (see Table 1) since it generally reduces the level of mesh adaptivity. However, since the mesh is smoother, larger stepsizes are taken during the course of time integration (see Figure 

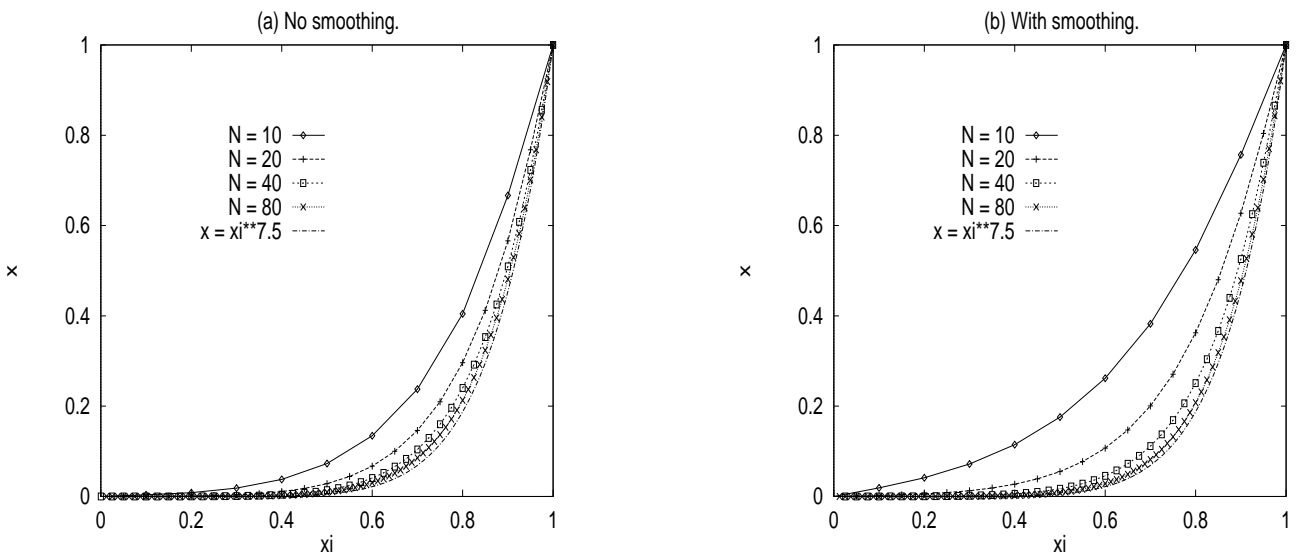

FIG. 2. Example 5.1. Meshes for different values of $N$.
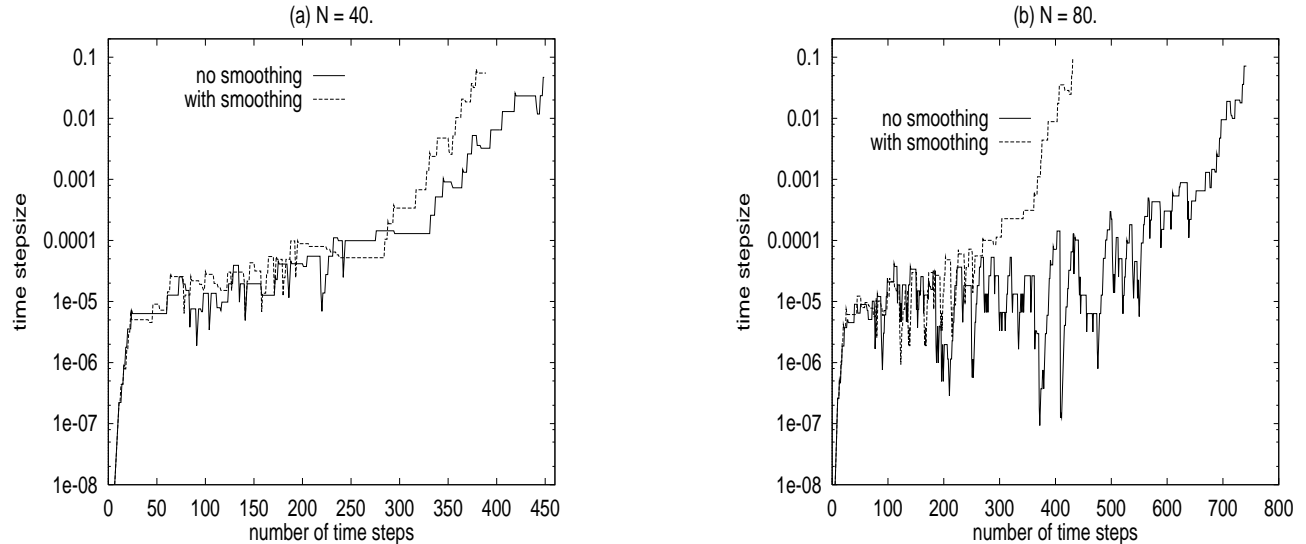

Fig. 3. Example 5.1. The time stepsize as function of time step for the cases $N=40$ and 80 .

3 ) and overall CPU time is reduced (see Table 1). With or without smoothing, the MMPDE produces very nonuniform meshes, e.g., $G(1)=6.94 \times 10^{6}$ and $6.74 \times 10^{5}$ when $N=80$ for the unsmoothed and smoothed cases, respectively.

Example 5.2. Burgers's equation with a smooth initial solution. Our second example is the well-known Burgers equation with a smooth initial solution,

$$
\begin{array}{ll}
u_{t}=\epsilon u_{x x}-\left(\frac{u^{2}}{2}\right)_{x}, & 0<x<1,0<t \leq 2, \epsilon=10^{-4}, \\
u(0, t)=u(1, t)=0, & 0<t \leq 2, \\
u(x, 0)=\sin (2 \pi x)+\frac{1}{2} \sin (\pi x), & 0 \leq x \leq 1 .
\end{array}
$$

This problem frequently serves as a test example for moving mesh methods, e.g., in [GDM81] and [FVZ90]. The solution is a wave that develops a very steep gradient and subsequently moves towards $x=1$. Because of the zero boundary values, the wave amplitude diminishes with increasing time. This is quite a challenging problem for methods which employ centered difference approximations. Proper placement of the fine mesh is critical, and these moving mesh methods tend to generate spurious oscillations as soon as the mesh becomes slightly too coarse in the layer region, just like standard centered differences with a nonmoving mesh. 
TABLE 2

Numerical results for Example 5.2 with $\epsilon=10^{-4} . L, G$, and $\|e\|_{1}$ are evaluated at $t=2$.

\begin{tabular}{ccccccc}
\hline \hline & $N$ & $L$ & $G$ & $\|e\|_{1}$ & Order & CPU (second) \\
\hline \hline No smoothing & 40 & 26.2 & $1.09 \mathrm{E} 3$ & $7.37 \mathrm{E}-3$ & & 32.5 \\
$\tilde{\gamma}=0$ & 50 & 31.1 & $1.08 \mathrm{E} 3$ & $5.77 \mathrm{E}-3$ & 1.10 & 47.7 \\
& 60 & 18.3 & $1.10 \mathrm{E} 3$ & $4.07 \mathrm{E}-3$ & 1.91 & 68.5 \\
& 70 & 20.6 & $1.10 \mathrm{E} 3$ & $3.44 \mathrm{E}-3$ & 1.09 & 87.1 \\
& 80 & 17.3 & $1.09 \mathrm{E} 3$ & $2.52 \mathrm{E}-3$ & 2.33 & 97.0 \\
& 100 & 12.7 & $1.06 \mathrm{E} 3$ & $1.46 \mathrm{E}-3$ & 2.45 & 1183.0 \\
\hline \hline With smoothing & 40 & 1.96 & $9.46 \mathrm{E} 2$ & $5.24 \mathrm{E}-4$ & & 9.58 \\
$\tilde{\gamma}=1$ & 50 & 1.95 & $9.69 \mathrm{E} 2$ & $2.62 \mathrm{E}-4$ & 3.11 & 13.4 \\
& 60 & 1.94 & $9.79 \mathrm{E} 2$ & $1.48 \mathrm{E}-4$ & 3.13 & 17.0 \\
& 70 & 1.93 & $9.85 \mathrm{E} 2$ & $1.08 \mathrm{E}-4$ & 2.04 & 20.1 \\
& 80 & 1.92 & $9.89 \mathrm{E} 2$ & $8.36 \mathrm{E}-5$ & 1.92 & 24.7 \\
& 100 & 1.91 & $9.93 \mathrm{E} 2$ & $4.40 \mathrm{E}-5$ & 2.88 & 35.9 \\
\hline \hline
\end{tabular}
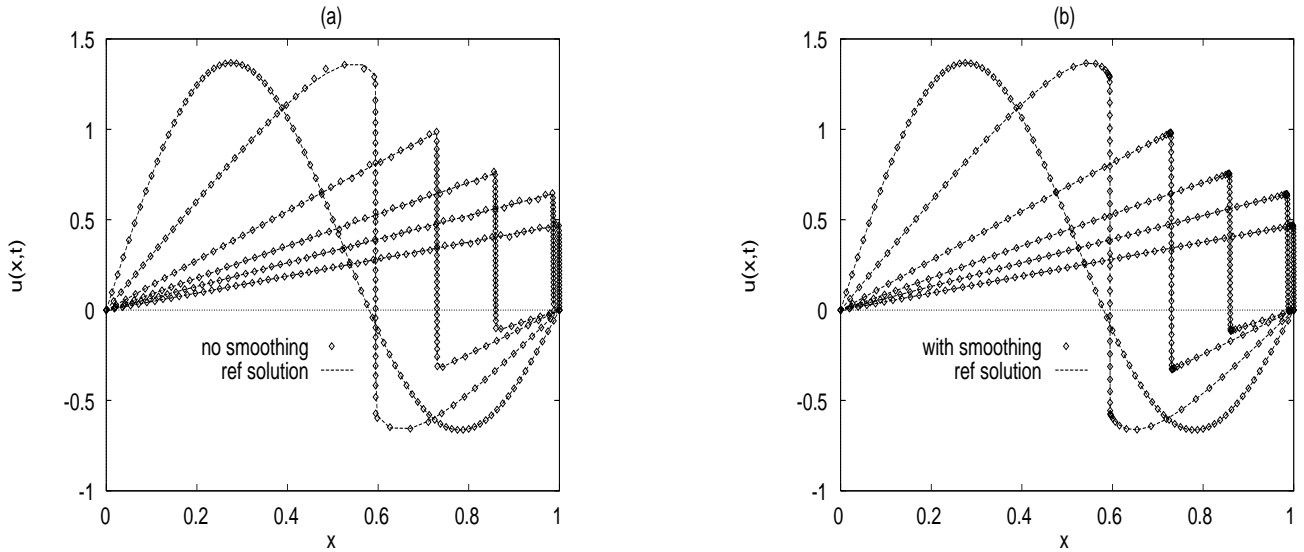

FiG. 4. Example 5.2. Solutions at different times $t=0.0,0.2,0.6,1.0,1.4$, and 2.0. $N=80$ is used.

Similar to Example 5.1 we can consider using an optimal monitor function. However, the semidiscrete system with such a monitor function is now more difficult to integrate in time because the solution here changes much more abruptly than the solution for Example 5.1. A detailed study of the relative merits of the curvature monitor function and the arclength monitor function

$$
M(x, t)=\sqrt{1+\left(\frac{\partial u}{\partial x}\right)^{2}}
$$

is made by Blom and Verwer [BV89], who find numerical difficulties using the higherderivative (curvature) monitor function. This is frequently the case when the solution changes rapidly, and we shall use (105).

Results obtained with MMPDE (25) are presented in Table 2 and Figures 4-6. Here, the $L_{1}$-norm $\|\bullet\|_{1}$ is used to measure the error in space. The reference solution is obtained by using a moving collocation method with cubic piecewise polynomials and 201 mesh nodes (see [HR96]).

We see from Table 2 that the moving mesh method has a second-order convergence rate both with and without spatial smoothing. But, unlike in the previous example, the MMPDE without smoothing produces a very nonsmooth mesh. This is clearly 

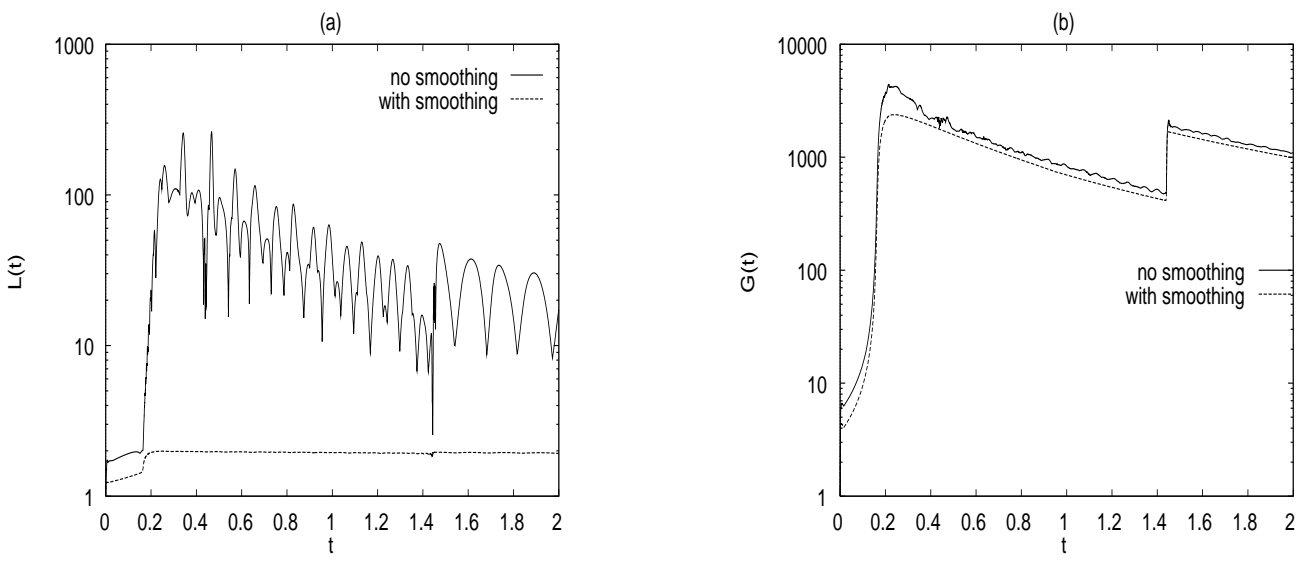

FiG. 5. Example 5.2. $L(t)$ and $G(t)$ are plotted as functions of time.
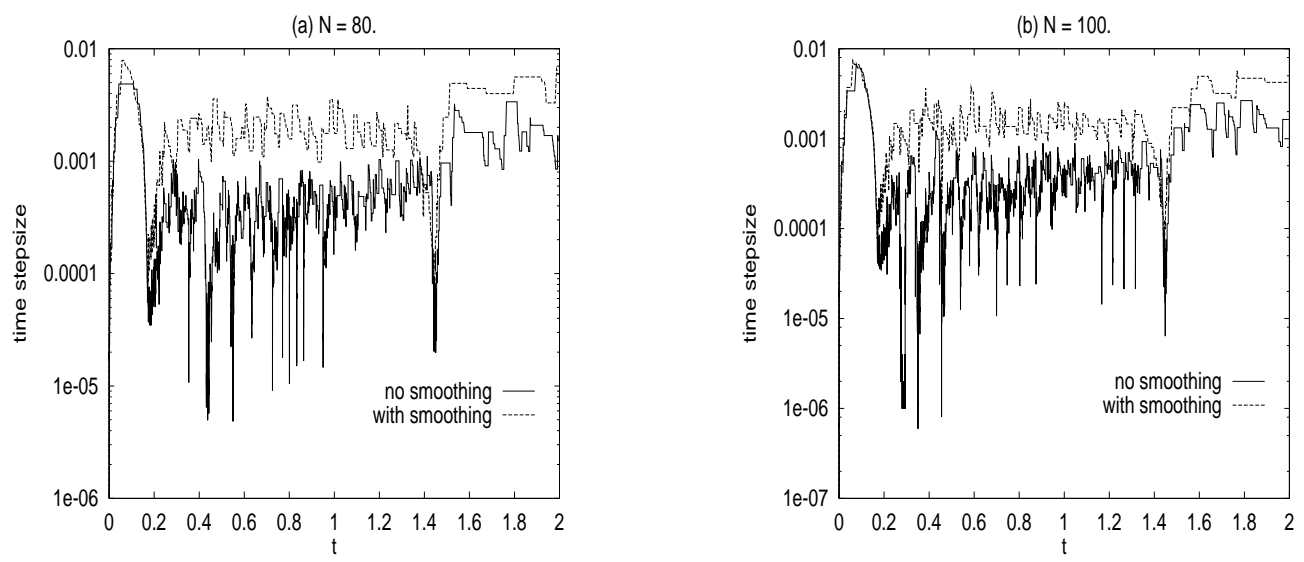

FIG. 6. Example 5.2. The time stepsize is plotted as a function of time.

seen from Table 2 and Figure 5(a), where $L(t)$ has a value about 17 at time $t=2$ and arrives at its maximal value of about 200 when the shock wave is formed. As a result of this, the accuracy decreases and very small time stepsizes are taken during the course of the time integration (see Figure 6). In fact, we can see from Figure 4(a) that there are still slight oscillations in the computed solutions when 80 subintervals are used.

On the other hand, the MMPDE with spatial smoothing produces much better results for this example. Table 2 and Figure 4 show clearly that it gives higher accuracy than the MMPDE without smoothing. Furthermore, much larger time stepsizes are taken during the time integration of the semidiscrete system with smoothing (see Figure 6), and the CPU time is much smaller (Table 2). From Theorem $4.1 \nu^{-1}=2$ for $\tilde{\gamma}=1$. Figure $5(\mathrm{a})$ and Table 2 show that $L(t)$ is bounded above by $\nu^{-1}$ for the MMPDE with smoothing, which is consistent with the conclusions of Theorem 4.1. Figure 5(b) clearly shows that with $\tilde{\gamma}=1$ the level of global quasi uniformity is very close to that without smoothing.

It is interesting to compare the performance of MMPDEs (19) and (25). The rapid initial increase in the time stepsize in Figure 6 illustrates the difficulty of starting 
the computation with MMPDE (25), and in fact stepsizes as small as about $10^{-9}$ are required initially. This difficulty is even more serious for MMPDE (19), and for corresponding parameter values DASSL fails to find $\dot{x}_{i}(0)$ and $\dot{u}_{i}(0)$. This is due to the fact that (19) is nonlinear in $\dot{x}$, and successful computation of $\dot{x}_{i}(0)$ and $\dot{u}_{i}(0)$ requires that the monitor function (or the initial physical solution) be sufficiently smooth in the computational coordinate. This smoothness requirement is less restrictive for MMPDE (25), which is linear in $\dot{x}$ (although even for (25) DASSL can fail to find the initial values of $\dot{x}$ and $\dot{u}$ for a steep initial physical solution when an initial uniform mesh is used). To reduce the difficulty in starting the integration, we can first generate an initial mesh equidistributed with respect to the initial solution (e.g., see [HRR94b]), and then MMPDE (19) works quite well. Indeed, in this case MMPDEs (19) and (25) produce almost identical results. Moreover, both faithfully produce locally quasiuniform meshes.

6. Conclusions and comments. In [HRR94a] and [HRR94b] we analyze and use moving mesh methods based upon solving MMPDEs which implicitly incorporate a temporal smoothing of the mesh transformation. Computational experience also indicates that if solutions to the underlying PDEs undergo rapid change, corresponding behavior of the monitor function results in the need for some sort of solution smoothing.

Here, we introduce a general method for smoothing $M$ which is basically used in [HRR94b]. It is based upon a familiar smoothing device in many contexts - the introduction of artificial diffusion. The resulting MMPDE (12) is rewritten in a form in which the smoothing is applied to the mesh transformation $x$ or concentration function $n$, and these MMPDEs (19) and (25) (with appropriate boundary conditions) are shown to satisfy the no node-crossing condition (34) and local quasi-uniformity condition (35). Discretizing (25) with three-point centered differences produces the well-known moving mesh scheme of Dorfi and Drury [DD87]. In section 4, an analogous analysis to that for the continuous problem is done. It shows that under mild assumptions one preserves the equivalents of (34) and (35). Thus, no node-crossings occur, and preservation of local quasi uniformity follows automatically without the need for a special mesh readjustment algorithm such as the one in [KN82].

While the analysis of the Dorfi-Drury method is similar to that in [VBFZ89], we emphasize that our approach is much more general. In particular, this method is only one of many possible discretizations of (25) (or (19) or (12)), and "smoothed" MMPDEs corresponding to the other MMPDEs in [HRR94a] lead to still other classes of methods which bear investigating. Also, this framework allows for the natural construction and analysis of MMPDEs in higher dimensions which use smoothing, as we are currently investigating. Finally, artificial diffusion to smooth meshes and the tools introduced here for analyzing its effect are applicable in other adaptive mesh selection contexts as well (e.g., see [HN84] and [BS82], where artificial diffusion is used to smooth the mesh during static rezoning).

Our numerical experiments here (and elsewhere) indicate that spatial smoothing is often an essential feature of an overall adaptive mesh selection procedure if the solution to the physical PDE rapidly changes. Even when this is not the situation, the numerical results do not appear to deteriorate significantly with the use of smoothing. Such is the case for the problems having derivative singularities considered by Gui and Babuška [GB86], for which we show also how the optimal meshes can be constructed using the MMPDEs with suitable monitor functions. 


\section{REFERENCES}

[AF86] S. Adjerid And J. E. Flaherty, A moving finite element method with error estimation and refinement for one-dimensional time dependent partial differential equations, SIAM J. Numer. Anal., 23 (1986), pp. 778-795.

[BS82] J. U. Brackbill and J. S. Saltzman, Adaptive zoning for singular problems in two dimensions, J. Comput. Phys., 46 (1982), pp. 342-368.

[BV89] J. G. Blom And J. G. Verwer, On the Use of the Arclength and Curvature Monitor in a Moving Grid Method which is Based on the Method of Lines, Tech. report NM-N8902, CWI, Amsterdam, 1989.

[CD85] G. F. CAREY AND H. T. Dinh, Grading functions and mesh redistribution, SIAM J. Numer. Anal., 22 (1985), pp. 1028-1040.

[DD87] E. A. Dorfi AND L. O'C. DruRY, Simple adaptive grids for 1-D initial value problems, J. Comput. Phys., 69 (1987), pp. 175-195.

[FVZ90] R. M. Furzeland, J. G. Verwer, And P. A. Zegeling, A numerical study of three moving grid methods for one-dimensional partial differential equations which are based on the method of lines, J. Comput. Phys., 89 (1990), pp. 349-388.

[GB86] W. GUI AND I. BABUS̆KA, The $h, p$ and $h-p$ versions of the finite element method in one dimension, Part 1: The error analysis of the p-version; Part 2: The error analysis of the $h$ and $h-p$ version; Part 3: The adaptive $h-p$ version, Numer. Math., 40 (1986), pp. 577-612, pp. 613-657, pp. 659-683.

[GDM81] R. J. Gelinas, S. K. Doss, And K. Miller, The moving finite element method: application to general partial differential equations with multiple large gradients, J. Comput. Phys., 40 (1981), pp. 202-249.

[HGH91] D. F. Hawken, J. J. Gottlieb, And J. S. HAnsen, Review of some adaptive nodemovement techniques in finite element and finite difference solutions of PDEs, J. Comput. Phys., 95 (1991), pp. 254-302.

[HN84] J. M. Hyman And M. J. NAUGhton, Static rezone methods for tensor-product grids, in Proc. SIAM-AMS Conference on Large Scale Computation in Fluid Mechanics, SIAM, Philadelphia, PA, 1984.

[HR96] W. HuAng ANd R. D. Russell, A moving collocation method for the numerical solution of time dependent partial differential equations, Appl. Numer. Math., 20 (1996), pp. 101-116.

[HRR94a] W. Huang, Y. Ren, And R. D. Russell, Moving mesh partial differential equations (MMPDEs) based on the equidistribution principle, SIAM J. Numer. Anal., 31 (1994), pp. 709-730.

[HRR94b] W. Huang, Y. Ren, And R. D. Russell, Moving mesh methods based upon moving mesh partial differential equations, J. Comput. Phys., 113 (1994), pp. 279-290.

[KN82] J. Kautsky and N. K. Nichols, Smooth regrading of discretized data, SIAM J. Sci. Statist. Comput., 3 (1982), pp. 145-159.

[MTW85] C. W. Mastin, J. F. Thompson, And Z. U. A. Warsi, Numerical Grid Generation: Foundations and Applications, Elsevier, New York, North-Holland, Amsterdam, 1985.

[Pet82] L. R. Petzold, A Description of DASSL: A Differential/Algebraic System Solver, Tech. report SAND82-8637, Sandia Labs, Livermore, CA, 1982.

[SHR96] W. Sun, W. HuAng, AND R. D. Russell, Finite difference preconditioning for solving orthogonal collocation equations for boundary value problems, SIAM J. Numer. Anal., 33 (1996), pp. 2268-2285.

[VBFz89] J. G. Verwer, J. G. Blom, R. M. Furzeland, and P. A. Zegeling, A moving grid method for one-dimensional PDEs based on the method of lines, in Adaptive Methods for Partial Differential Equations, J. E. Flaherty, P. J. Paslow, M. S. Shephard, and J. D. Vasilakis, eds., SIAM, Philadelphia, PA, 1989, pp. 160-175.

[VR92] A. E. P. Veldmon And K. Rinzema, Playing with nonuniform grids, J. Engrg. Math., 26 (1992), p. 119. 